\title{
Newly learned shape-colour associations show signatures of reliability- weighted averaging without forced fusion or a memory colour effect
} \author{
Nardini ${ }^{1}$ \\ ${ }^{1}$ Department of Psychology, Durham University, UK \\ ${ }^{2}$ Biosciences Institute, Newcastle University, UK \\ *stacey.j.aston@durham.ac.uk
}

Stacey Aston ${ }^{1 *}$, Cat Pattie ${ }^{2}$, Rachael Graham ${ }^{1}$, Heather Slater ${ }^{1}$, Ulrik Beierholm $^{1}$, \& Marko

\begin{abstract}
Reliability-weighted averaging of multiple perceptual estimates (or cues) can improve precision. Research suggests that newly-learned statistical associations can be rapidly integrated in this way for efficient decision-making. Yet, it remains unclear if integration of newly-learned statistics into decision-making can directly influence perception, rather than taking place only at a decision stage. In two experiments, we implicitly taught observers novel associations between shape and colour. Observers made colour matches by adjusting the colour of an oval to match a simultaneously presented reference. As the colour of the oval changed across trials, so did its shape according to a novel mapping of axis ratio to colour. Observers showed signatures of reliability-weighted averaging - a precision-improvement in both experiments and reweighting of the newly-learned shape cue with changes in uncertainty in Experiment 2. To ask whether this was accompanied by perceptual effects, Experiment 1 tested for "forced fusion" by measuring colour discrimination thresholds with and without incongruent novel cues. Experiment 2 tested for a "memory colour effect", observers adjusting the colour of ovals with different axis ratios until they appeared grey. There was no evidence for forced fusion and the opposite of a memory colour effect. Overall, our results suggest that the ability to quickly learn novel cues and integrate them with familiar cues is not immediately (within the short duration of our experiments) accompanied by common perceptual effects.
\end{abstract}

\section{Introduction}

Reliability-weighted averaging of multiple perceptual estimates (or cues) has been shown across a variety of perceptual domains for human observers (Landy et al., 2011). For example, cues to depth are combined according to their reliabilities both across and within sensory modalities (Ernst \& Banks, 2002; Hillis et al., 2002, 2004; Jacobs, 1999; Knill \& Saunders, 2003; Nardini et al., 2010). Combining independent noisy estimates in this way is the optimal strategy for maximising perceptual precision, as the resulting estimate is less variable (more reliable) than estimates from either single cue alone (Jones, 2016a; Rohde et al., 2016a). 
Previous work shows that newly-learned novel cues can also be combined with familiar cues to make more precise decisions (Aston, Beierholm, et al., 2021; Ernst, 2007; Gibo et al., 2017; Negen et al., 2018 , 2021). For example, a newly-learned audio cue to depth is combined with visual information to improve precision in depth judgements after short-term training (Negen et al., 2018, 2021). Similarly, along another dimension of spatial localisation, novel visual cues are combined with familiar cues to increase the precision of horizontal location estimates (Aston, Beierholm, et al., 2021). However, it remains unclear if novel cues have the same direct influence on perception as familiar cues or if they only influence final decision-making. For example, multiple familiar cues are subject to a phenomenon referred to as forced fusion, where the perceptual system cannot help but take a reliability-weighted average of the cues that directly alters perception, even if keeping the cues separate and making a decision based on appearance due to each single cue alone would lead to better discrimination (Hillis et al., 2002; Nardini et al., 2010).

Here, we extend the limited work on efficient use of novel cues to the domain of material perception. We test participants in a short-term training study with a novel shape-colour association, making object shape a useful cue to object colour within the task. We test whether perceptual precision with the addition of this new cue, and further, we test whether any such abilities are accompanied by perceptual effects. To be clear, we define a perceptual effect as one where how the object appears to the participant changes based on the new cue, rather than the new cue only influencing final decision-making.

We introduce a novel shape-colour association (a novel shape cue to colour) by creating an arbitrary association between the axis ratio of an oval and its colour. In each experiment, following a period of repeated exposure to the novel shape-colour association, we tested for the two key signatures of reliability-weighted averaging that are commonly tested for in the cue combination literature: (1) a reduction in colour matching variability when the shape cue is present and (2) re-weighting of the shape cue with change in familiar cue uncertainty (Ernst \& Banks, 2002; Jones, 2016b; Rohde et al., 2016b). We also tested for a perceptual effect of the novel shape cue to colour.

In summary, we found signatures of reliability-weighted averaging of the novel shape cue and familiar cues to colour in both experiments. Participants showed a reduction in colour matching variability in both experiments and cue reweighting with changes in uncertainty in Experiment 2. However, our results overall suggest that the ability to quickly learn novel cues and take a reliabilityweighted average of novel and familiar cues is not immediately (within the short duration of our experiments) accompanied by common perceptual effects.

\section{Experiment 1}


In Experiment 1 we introduce a novel shape cue to colour by creating an arbitrary association between the axis ratio of an oval and its colour (see Figure 1). The direction of the association was varied across observers. For some participants, a taller oval mapped to a pinkish colour and a wider oval mapped to a greenish colour. For others, the mapping was reversed.

To teach observers the novel relationship between shape and colour, the experiment began with an exposure block, where participants were exposed to the novel relationship. On each trial in this block, participants were presented with a reference patch whose axis ratio was defined by the novel mapping from shape to colour, and a matching patch with the same axis ratio as the reference patch. Participants were required to adjust the colour of the matching patch to match that of the reference patch by moving a cursor controlled by the mouse along a colour gradient. Following the exposure block, we tested for (1) a reduction in matching variability when the novel cue was present in the matching patch compared to when it was absent (removing the novel cue by making the matching patch square), and (2) changing novel cue weights with changing familiar cue uncertainty. We increased familiar cue uncertainty by adding a delay between presentation of the reference and matching patches.

We tested for a perceptual effect of the novel shape cue to colour in two ways. Firstly, by including a simultaneous matching condition (the low uncertainty condition) where the novel cue was always present in the reference patch but could be present or absent in the matching patch. In this simultaneous matching condition, regardless of whether the novel cue is present or absent in the matching patch, the same information is available to the participants to complete the task (both familiar and novel cues to colour are present on the screen). We propose that a reduction in matching variability when the novel shape cue is present in the matching patch (compared to when it is absent) in the simultaneous matching condition points towards a perceptual effect of the novel cue. The logic here is that if matching variability is reduced only when the novel cue is present in the matching patch, even though the same information is available in the reference patch when the novel cue is absent from the matching patch, then the novel shape cue has a perceptual effect on the appearance colour of the matching patch, reducing appearance colour variability when present and, subsequently, matching variability.

We also tested for a perceptual effect by testing for forced fusion, a feature of familiar cue integration (Hillis et al., 2002; Nardini et al., 2010). An ideal decision-maker considering the evidence from both cues would not be subject to fusion, but one who has no access to the single cues but only to a combined percept would be subject to fusion. We therefore consider fusion to be diagnostic of a potentially perceptual effect, where the observer comes to perceive the combined percept and not 
its constituent cues. To test for forced fusion, participants completed a two-alternative forced choice (2AFC) task. In the task, one of the two alternatives was an exact match to the reference. The other alternative was offset from the reference in both shape and colour, but the offsets were opposing such that an equal weighting of familiar and novel cues would produce a matching colour to the reference. We also manipulated whether the novel cue was present or absent to establish baseline thresholds for discrimination. Under the assumption of forced fusion, we expected thresholds to be higher when the novel cue was present as conflicting information would make the two alternatives appear more similar.

\section{Methods}

\section{Participants}

We recruited 35 participants (30 female, age range 18-35 years) to take part in Experiment 1 . One was excluded as they failed to meet our inclusion criteria (see Exclusion Criteria) leaving 34 participants in the analysis. Participants were recruited through word of mouth, our department's undergraduate participant pool, and our lab's Facebook page. Before they could take part in the experiment, participants were screened for colour vision deficiencies using the Ishihara plates. All participants were compensated with $£ 10$ for their time plus an additional bonus of $£ 5-10$ depending on performance. If participants got $X \%$ of the total points available, they received $f(X+10) / 10$ bonus, rounded down, but received a minimum of $£ 5$.

\section{Ethics}

Ethical approval was received from the Durham University Psychology Department Ethics Board (reference number: 17/07). All participants gave written, informed consent prior to taking part in the experiment.

\section{Apparatus}

Stimuli were shown on a 10-bit ASUS Proart LCD screen (ASUS, Fremont, CA) in a dark room (no light source except the screen) with participants seated so that their eyes were approximately $60 \mathrm{~cm}$ from the screen. The monitor was controlled using a 64-bit Windows machine, equipped with an NVIDIA Quadro K600 10-bit graphics card (NVIDIA, Santa Clara, CA), running MATLAB scripts that used Psychtoolbox routines (Brainard, 1997; Kleiner et al., 2007; Pelli, 1997). The stimuli were colourimetrically calibrated using a linearized calibration table based on measurements of the monitor primaries made with a Konica Minolta CS2000 spectroradiometer (Konica Minolta, Nieuwegein, Netherlands). Conversions to CIELUV used the measured white point of the monitor: $(Y, x, y)=(205.24, .31, .34)$ in CIE 1931 Yxy colour space. 


\section{Stimuli}

All colours used in the experiment were parameterised to fall along a gradient from pinkish to greenish. The gradient was defined as a chord of a hue circle (chroma $=85$ ) in CIELUV chromaticity space. The start and end values of the chord had CIE 1931 chromaticities of $(x, y)=$ $(.3386, .2821)$ and $(x, y)=(.3476, .3960)$ and a luminance of $Y=15 \mathrm{~cd} / \mathrm{m}^{2}$. The colour gradient was defined in this way to ensure perceptual uniformity. In other words, equal distances along the gradient should correspond to roughly equal perceptual differences. We arbitrarily assign a value of 0 to one end of the colour gradient and 1 to the other. Then, all colours along the gradient can be specified by a number between 0 and 1 . The end of the gradient that was assigned a value of 0 was decided at random for each participant.

The stimuli shown during the experiment were coloured ovals (ellipses) in the colours described above (Figure 1A). During the experiment, we created a novel association between the axis ratios (vertical/horizontal) of oval and their colour. The axis ratio varied from 0.33 to 3 , with the minimum and maximum axis lengths being 3 and $9 \%$ of the screen width, respectively. Importantly, as the axis ratio changed, the area of the oval was kept the same. The smallest and largest axis ratios were each identified with one of the arbitrary colour values 0 or 1 across the colour gradient. The smallest axis ratio was identified with either 0 or 1 at random for each participant. Thus, all colours could be identified with a unique axis ratio and, as the area was maintained, unique lengths for the vertical and horizontal axes. Each participant was exposed to one of the two possible mappings between colour and shape, and we randomly determined which mapping each participant saw (Figure 1A). 

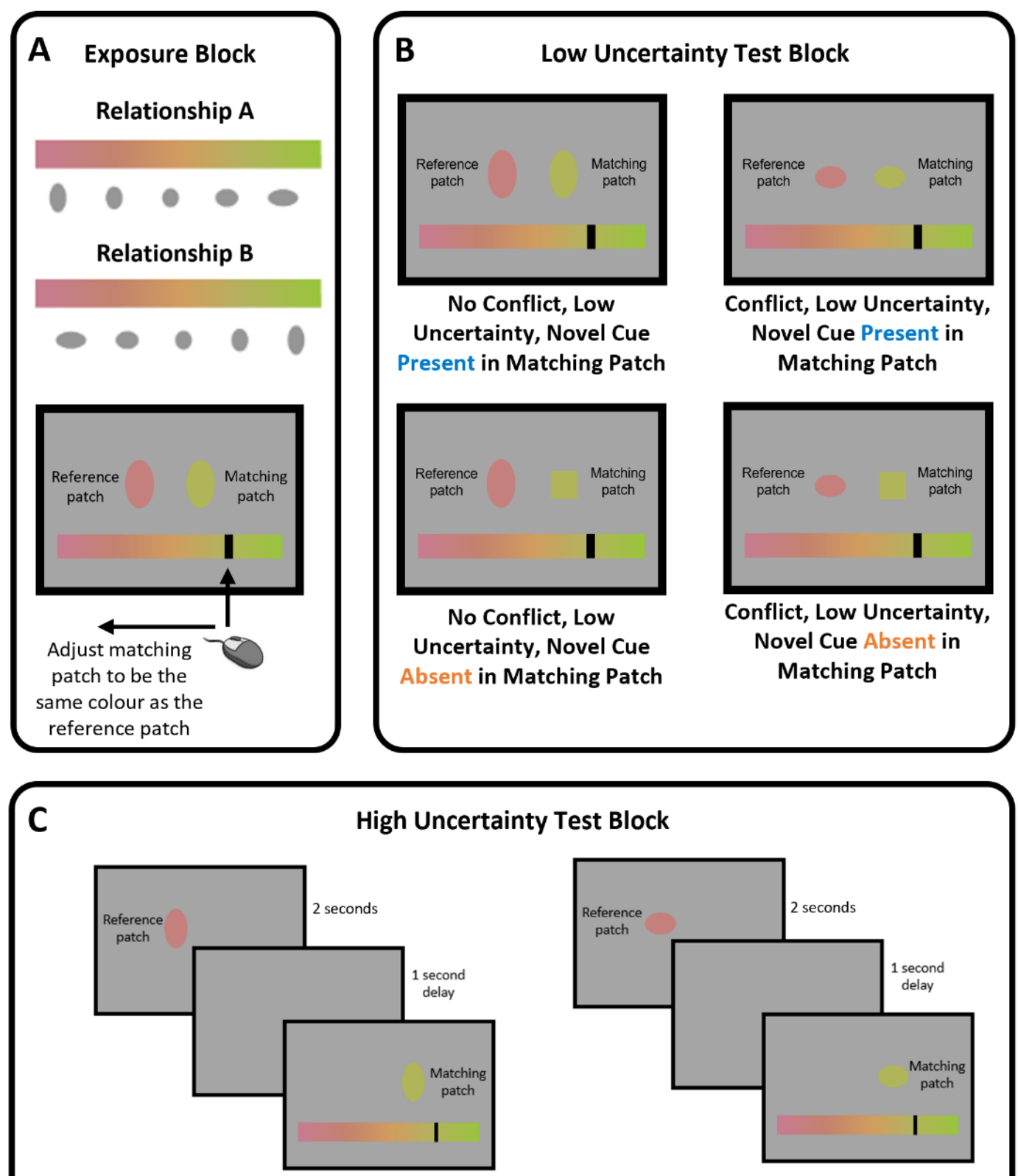

No Conflict, High Uncertainty, Novel Cue Present in Matching Patch

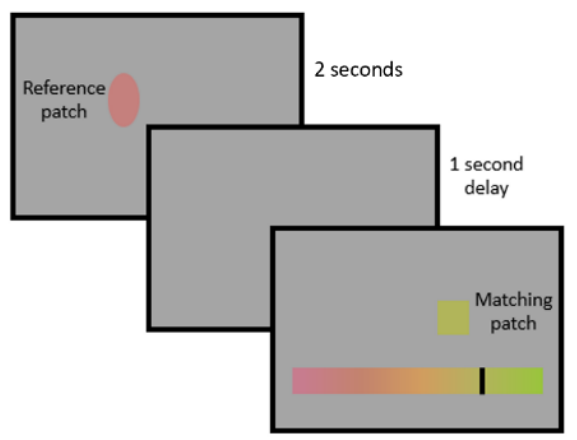

No Conflict, High Uncertainty, Novel Cue Absent in Matching Patch

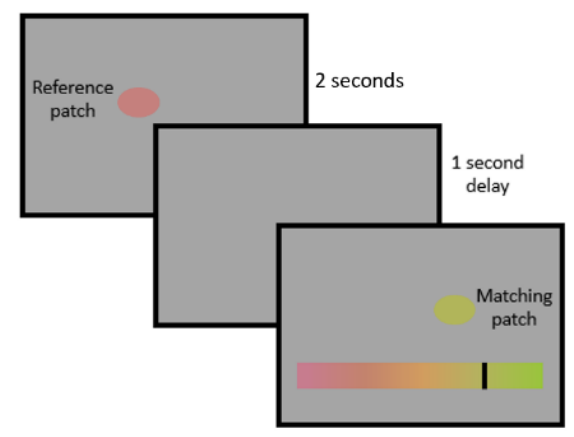

Conflict, High Uncertainty, Novel Cue Present in Matching Patch

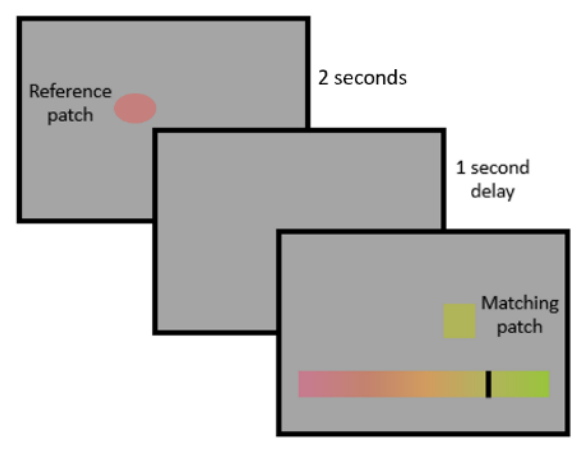

Conflict, High Uncertainty, Novel Cue Absent in Matching Patch 
Figure 1: Adjustment trials in Experiment 1. (A) The experiment began with an exposure block where, through repeated simultaneous colour matches, participants could learn the novel shape-colour relationship. Participants learnt relationship $A$ or $B$ at random. (B) In the low uncertainty test block participants also completed simultaneous colour matches, but the trials were now one of four types, varying whether the novel shape cue was present in the matching patch and whether the novel shape cue conflicted with the colour of the reference patch according to the novel relationship introduced in the exposure block. (C) In the high uncertainty test block participants completed successive colour matches, with the reference patch shown for 2 seconds and a delay of 1 second before presentation of the matching patch. Again, there were four types of trials in this block, varying novel cue presence in the matching patch and conflict between familiar and novel cues.

\section{Procedure}

There were five blocks of trials in the task: the exposure block (simultaneous colour matching; block 1), the low uncertainty test block (simultaneous colour matching; block 2), the top-up exposure block (simultaneous colour matching; block 3), the high uncertainty test block (successive colour matching; block 4), and the forced fusion block (2AFC; block 5). Twenty-seven participants completed the blocks in the order 1 to 5 . We had intended to switch the ordering of blocks 2 and 4 at random but did not catch the error in the MATLAB code that meant all participants saw the same order until deep into data collection. At that point we edited the code and the last 8 participants recruited completed the blocks in the order 1-4-3-2-5. All participants completed testing within a single session, typically lasting between 1.5 and 2 hours.

Blocks 1 to 4 consisted of adjustment trials, where participants could adjust the colour of one patch (the matching patch) to match the colour of another patch (the reference patch). There were eight types of adjustment trials as the shape of the matching patch, the delay between the reference and matching patch, and whether the familiar and novel cues were in conflict could vary. These were important manipulations for addressing our research questions. The shape of the reference patch (oval or square) manipulated the presence of the novel shape cue in the matching patch (novel cue present or absent). The delay between presentation of the reference and matching patch (no delay or 1 second delay) manipulated the uncertainty of familiar (low-level) cues to colour (low or high familiar cue uncertainty). Adding conflict between the familiar cues to colour and novel shape cue to colour allowed us to measure the amount of weight that participants placed on the novel cue.

Blocks 1-4 were made up of different combinations of these trials, as detailed below. The choice block was a 2AFC task. In these trials, participants were presented with a reference patch and two comparisons. They had to select the comparison that they perceived as the best colour match to the reference.

During the exposure block (Figure 1A), adjustment trials were all the same type: no conflict, low uncertainty trials with the novel cue present in the matching patch. Participants were 
simultaneously presented with the reference patch (on the left of the screen) and the matching patch (on the right of the screen). The colour of the reference patch had a value between 0.05 and 0.95 on our $0-1$ reference scale (see above) in steps of 0.05 , each shown 10 times -190 trials in total. Both the reference and matching patch were ovals with the axis ratio that corresponded to the colour value of the reference patch. The starting colour of the matching patch was a random value between 0 and 1 . At the bottom of the screen, participants saw the whole colour gradient as a horizontal bar. By moving the mouse, they could move a black vertical line left and right across the gradient, changing the colour of the matching patch, and left click to issue a response. After responding, participants received feedback gaining several points for that trial. Points were awarded according to a mean squared error loss function.

Adjustment trials in the low uncertainty test block were the same as in the exposure block, except that the matching patch could be a square on some trials and/or the axis ratio of the oval could conflict with the corresponding colour (Figure 1B). Thus, there were four types of trials in this block: (1) no conflict, low uncertainty trials with the novel cue present in the matching patch; (2) no conflict, low uncertainty trials with the novel cue absent in the matching patch; (3) conflicting, low uncertainty trials with the novel cue present in the matching patch; and (4) conflicting, low uncertainty trials with the novel cue absent in the matching patch. For trials where there was no conflict, regardless of whether the novel cue was present or absent in the matching patch, the colour of the reference patch had an arbitrary value between 0.05 and 0.95 in steps of 0.1 , each repeated 5 times for each trial type. In trials without conflict and where the novel cue was present in the matching patch, the reference and matching shape were ovals with axis ratios corresponding to the colour of the reference patch. In trials without conflict and where the novel cue was absent from the matching patch, the matching patch was a square with side length $5.2 \%$ of the screen width. For the trials with conflict, regardless of whether the novel cue was present or absent in the matching patch, the colour of the reference patch had an arbitrary value of 0.25 or 0.75 , but the axis ratio of the reference oval conflicted with this value by $-10,-5,5$, or $10 \%$ of the mapping. In trials with conflict and where the novel cue was present in the matching patch, the matching patch was also an oval with the conflicting value. In trials with conflict and where the novel cue was absent from the matching patch, the matching patch was a square with side length $5.2 \%$ of the screen width. There were five repeats of each reference and conflict value pairing for each trial type and a total of 180 trials in this block in total.

The top-up exposure block was identical to the exposure block (Figure $1 \mathrm{~A}$ ), containing only no conflict, low uncertainty trials with the novel cue present in the matching patch, except that the number of repeats for each reference was reduced to 3 for 57 trials in this block in total. 
The high uncertainty test block was identical to the low uncertainty test block except that the reference patch was now only shown for 2 seconds, and participants were only allowed to issue their response after a 1 second delay (Figure 1C). Hence, this block also contained four types of trials: (1) no conflict, high uncertainty trials with the novel cue present in the matching patch; (2) no conflict, high uncertainty trials with the novel cue absent in the matching patch; (3) conflicting, high uncertainty trials with the novel cue present in the matching patch; and (4) conflicting, high uncertainty trials with the novel cue absent in the matching patch.

The forced fusion block (Figure 2) was designed to test for forced fusion of the newly-learned novel shape cue and familiar cues to colour. To test for forced fusion in this block participants completed a 2AFC task. In the task one of the two alternatives was an exact match to the reference. The other alternative was offset from the reference in both shape and colour, but the offsets were opposing such that an equal weighting of familiar and novel cues would produce a matching colour to the reference. We also manipulated whether the novel cue was present or absent to establish baseline thresholds for discrimination. Under the assumption of forced fusion, we expected thresholds to be higher when the novel cue was present as conflicting information should make the two alternatives appear more similar.

Trials in the forced fusion block were split into two sections, one containing low uncertainty twoalternative forced choice trials and the other containing high uncertainty $2 \mathrm{AFC}$ trials. Whether the low or high uncertainty trials came first was randomised for each participant.

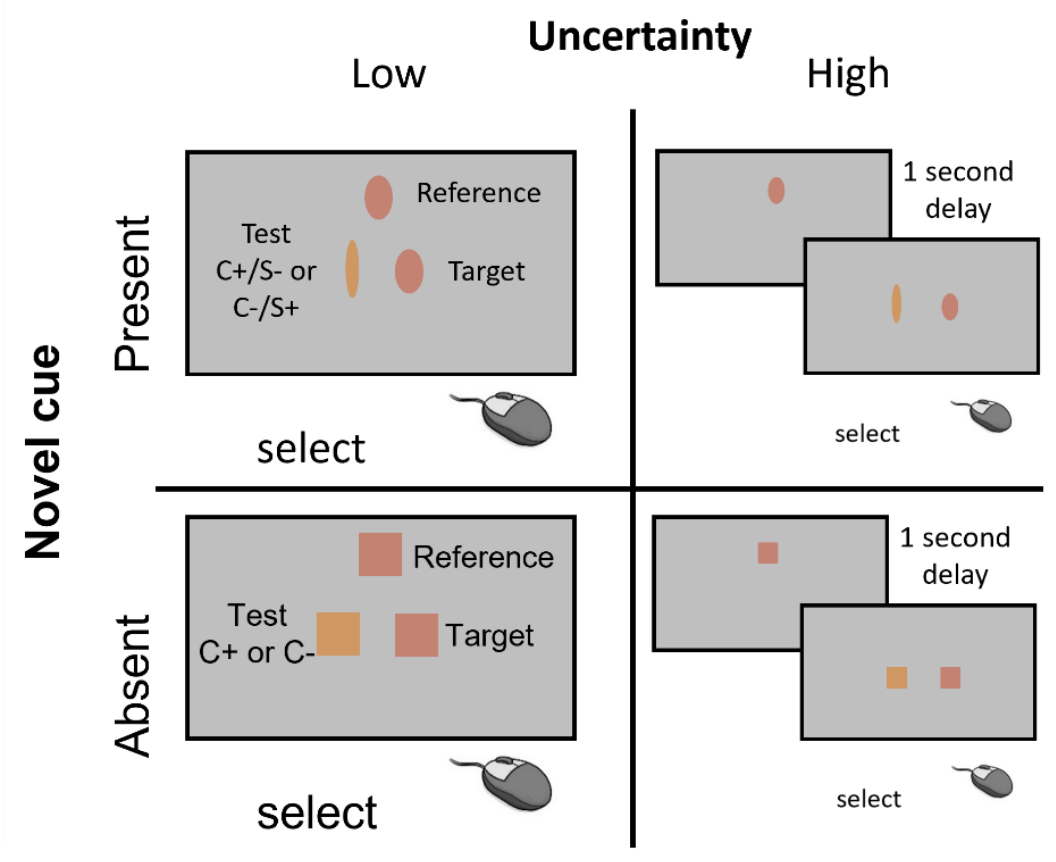


Figure 2: Forced fusion trials in Experiment 1. This part of the experiment consisted of two-alternative forced-choice trials that could be one of four types. The four trial types were defined by two varying factors: novel cue presence and familiar cue uncertainty. We varied novel cue presence by making reference and comparisons (the test and target patches) ovals, with axis ratios defined by the novel shape-colour relationship (novel cue present), or squares (novel cue absent). We varied familiar cue uncertainty by either presenting the reference and comparison patches simultaneously or adding a 1 second delay between presentation of the reference patch and the comparisons.

In the low uncertainty 2AFC trials participants were presented with a reference patch of colour at the top of the screen and two comparisons below it. In some trials the novel shape cue was present (novel present, low uncertainty 2AFC trials) and in others it was absent (novel absent, low uncertainty $2 \mathrm{AFC}$ trials). In novel absent, low uncertainty $2 \mathrm{AFC}$ trials, the reference and comparison patches of colour were all squares with side length $5.2 \%$ of the screen width. One of the comparisons was a perfect colour match to the reference (the target), the other varied from the reference by a different amount on each trial (the test), determined by a 1-up, 1-down staircase procedure with a step size of 0.02 that terminated after 35 trials or 15 reversals. The staircase procedure was used to establish $50 \%$ discrimination thresholds around each of two reference values: 0.25 and 0.75 . One staircase approached each reference from either direction with starting differences between the reference and test patches of -0.1 or 0.1 . Thus, there were four novel absent, low uncertainty staircases. These staircases established baseline $50 \%$ colour discrimination thresholds for each reference and in each direction along the colour gradient.

There were also four novel present, low uncertainty staircases made up of novel present, low uncertainty $2 \mathrm{AFC}$ trials. These trials/staircases were identical to the novel absent, low uncertainty trials/staircases except that all patches were ovals. The reference and target patches had axis ratios determined by the novel mapping from their colour to oval axis ratio, but the axis ratio of the test patch conflicted with its colour. If the difference between the colour of the reference and test patch was $X$, the difference between the axis ratio of the reference and the test patch was $-X$.

Subsequently, if the colour and shape cues were fused, thresholds should be larger when the novel cue is present as the test and match patches should appear more similar. All novel absent and novel present, low uncertainty staircases were interleaved so that there were eight staircases running simultaneously in this section of the forced fusion block.

There was a corresponding high uncertainty 2AFC trial or staircase for each type of low uncertainty 2AFC trial or staircase. The only difference between low and high uncertainty $2 \mathrm{AFC}$ trials was that in the high uncertainty $2 A F C$ trials, the reference patch was shown alone for two seconds and then there was a 1 second delay (where a blank grey screen was shown) before presentation of the two 
comparisons. The eight high uncertainty staircases were interleaved in a different section of the forced fusion block.

We only report data from the forced fusion block for 30 of the 35 participants. After the first four participants we refined the code so that it did not allow for the sign of conflicts to change, effectively preventing staircases from crossing over, something we did not consider until we saw it happen in a data set. One further participant was excluded after reporting to the experimenter that they were trying to pick the comparison that did not match the reference rather than the one that did.

\section{Data Analysis}

All calculations were performed in terms of screen proportions, and we calculated three different types of measures in this experiment. To test for signatures of reliability-weighted averaging we calculated matching variability (variable error) and the weight placed on the novel cue from different types of adjustment trials in blocks 2 and 4 . To test for a perceptual effect of the novel cue, we calculated discrimination thresholds from the staircases in the forced fusion block.

Matching variability was calculated for each type of no conflict adjustment trial in blocks 2 and 4: no conflict, low uncertainty trials with the novel cue present in the matching patch or the novel cue absent in the matching patch (block 2 ) and no conflict, high uncertainty trials with the novel cue present in the matching patch or the novel cue absent in the matching patch (block 4). Thus, we calculated matching variability for four types of adjustment trials.

To calculate matching variability we used a method described fully elsewhere (Aston, Negen, et al., 2021). In brief, the method is designed to account for central biases in continuous responses that may reduce statistical power for detecting a gain in precision using multiple cues. To calculate measures of variability according to the method, we regress matches for each trial type on the true colour value and calculate the standard deviation of the residuals. If the slope of the fitted regression line is significantly less than one, the standard deviation of the residuals is divided by the fitted slope of the regression line to correct for a central bias. If there is no evidence of a central bias (the slope is not significantly less than one), no correction is performed.

The weight placed on the novel cue was calculated for each of the four types of conflict adjustment trials in blocks 2 and 4: (1) conflicting, low uncertainty trials with the novel cue present in the matching patch (block 2); (2) conflicting, low uncertainty trials with the novel cue absent in the matching patch (block 2); (3) conflicting, high uncertainty trials with the novel cue present in the matching patch (block 4); and (4) conflicting, high uncertainty trials with the novel cue absent in the matching patch (block 4). 
To calculate the weight placed on the novel cue we again used a method that is described fully elsewhere (Aston, Negen, et al., 2021). In brief, we model matches, $m$, as

$$
m=\left(1-w_{c}\right) \times\left[w_{s} s+\left(1-w_{s}\right) f\right]+w_{c} \times 0.5+\epsilon,
$$

where $s$ is the value of the shape cue, $f$ is the value of low-level familiar cues to colour, $w_{S}$ is the weight on the shape cue, $w_{c}$ is the strength of the central bias (the central value being 0.5 ), and $\epsilon \sim$ $N\left(0, \sigma_{n}^{2}\right)$ is an additional noise term. We estimate the parameters $w_{s}, w_{c}$, and $\sigma_{n}$ using a Gibbs Sampler (JAGS; Plummer, 2003) implemented in MATLAB using the MATLAB-to-JAGS interface. We ran three independent chains, discarding the first 100 samples of each chain as burn-in, and recording 1000 samples after the burn-in period, thinned by recording only every 5th sample. Both fitted weights $\left(w_{s}\right.$ and $\left.w_{c}\right)$ were initialized at 0.5 in all chains. The standard deviation of the additional noise $\left(\sigma_{n}\right)$ was initialized at 0.01 . The resulting estimates were taken as the mean of the expected values from the three chains.

To calculate thresholds from the staircases in the forced fusion block we took the average of the last two reversals from each staircase.

\section{Exclusion criteria}

We planned to exclude participants from the analyses if their matching variability was too high for either of the low or high uncertainty conditions. Match variability had to be less than 0.075 for the low uncertainty condition and less than 0.15 for the high uncertainty condition.

\section{Results}

Matching variability reduced when the novel shape cue was present in the matching patch but only in the low uncertainty condition

Figure $3 \mathrm{~A}$ shows matching variability (variable error) in both the low (simultaneous matching) and high (successive matching) uncertainty conditions when the novel shape cue was present in the matching patch and when the novel shape cue was absent from the matching patch. Under the assumption of reliability-weighted averaging, we expected matching variability to be lower when the novel shape was present in the matching patch. In separate Wilcoxon Signed Rank tests, we found significantly lower matching variability when the novel cue was present in the low uncertainty condition $(z=-3.7, p<.001)$, but no difference between when the novel cue was present and when it was absent in the high uncertainty condition $(z=-0.44, p=.663)$. 
A

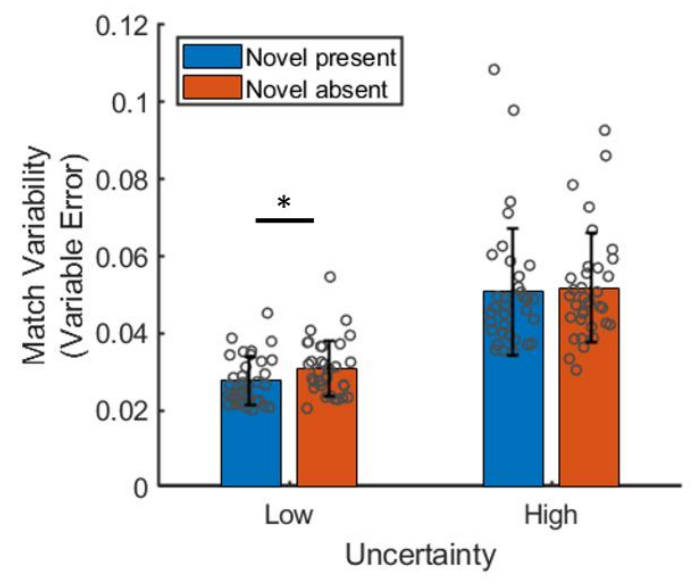

B

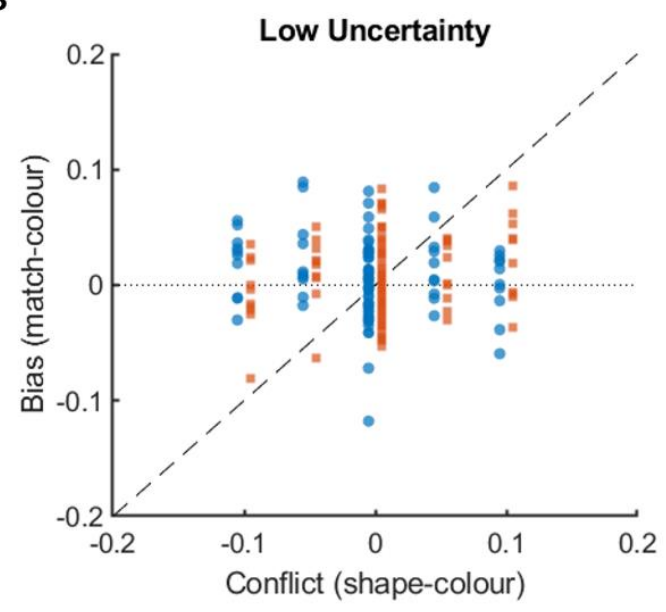

C

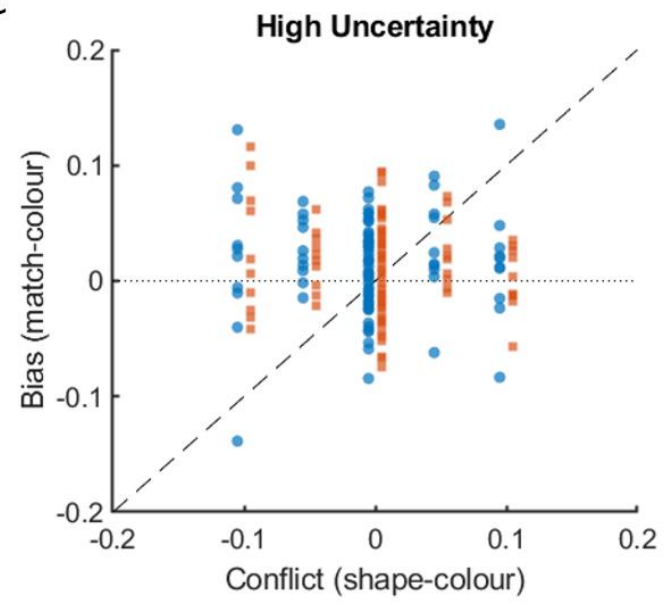

D

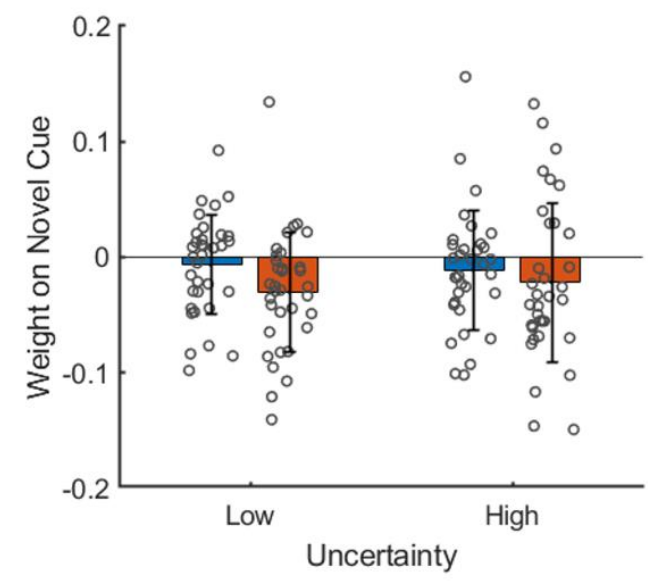

$\mathbf{E}$

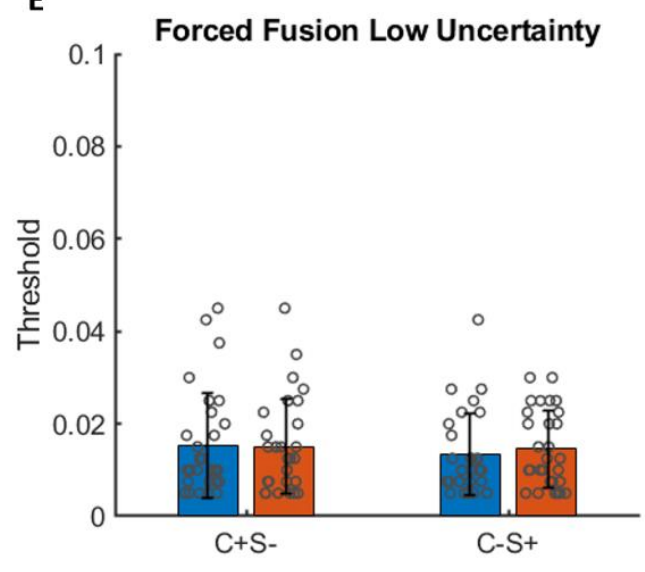

F

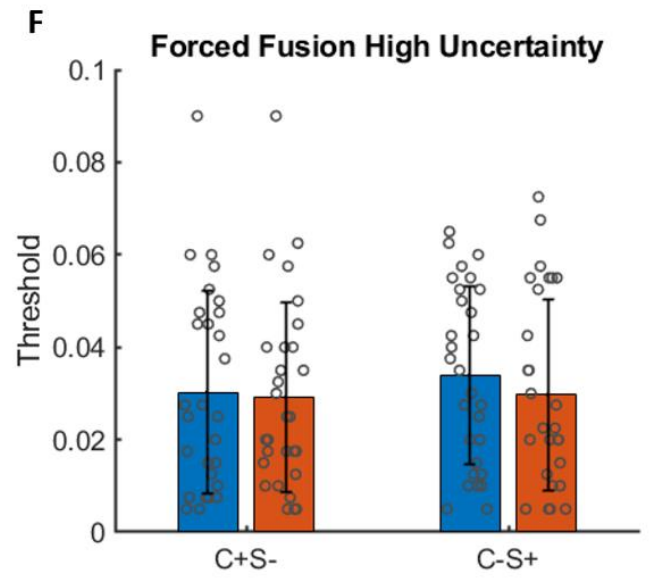

Figure 3: Experiment 1 results. (A) Matching variability (variable error) for each level of uncertainty split by novel cue presence. $(B / C)$ Individual matching biases (matched colour - reference colour) for an example participant plotted against the conflict between the shape (novel) and colour (familiar) cues in the reference patch (shape cue value - colour cue value) in the low $(B)$ and high $(C)$ uncertainty conditions. Blue dots are when the novel cue was present in the matching patch. Orange squares are when the novel cue was absent from the matching patch. (D) The inferred weight on the novel cue in each condition. (E/F) Discrimination thresholds from the forced fusion task for each condition. Error bars are +/- 1 SD. 
When novel and familiar cues conflicted, the weight on the novel cue did not differ significantly from zero regardless of novel cue presence in the matching patch or level of uncertainty

Figures $3 \mathrm{~B}$ and $\mathrm{BC}$ show data from an example participant on trials where the novel shape cue conflicted with familiar cues to colour. In these figures we plot the conflict (shape value - true colour value) on the horizontal axis and response bias (match value - true colour value) on the vertical axis. Blue dots are when the novel cue was present in the matching patch. Orange squares are when the novel cue was absent from the matching patch. Under the assumption of reliability-weighted averaging, conflicting novel shape cues should bias matches away from the true colour value and towards the conflicting novel shape cue value. These figures suggest this was not the case for this participant, who placed approximately zero weight on the novel shape cue regardless of whether the novel shape cue was present or absent in the matching patch. Indeed, when we fit the model described in the data analysis section to each participant's data individually, we find that the fitted weights on the novel shape cue, $w_{S}$, are not significantly greater than zero in either uncertainty condition, regardless of whether the novel cue was present or absent (novel absent, low uncertainty: $\bar{x}=-0.03, t(33)=-3.42, p=.999$; novel absent, high uncertainty: $\bar{x}=$ $-0.02, t(33)=-1.84, p=.962$; novel present, low uncertainty: $\bar{x}=-0.01, t(33)=-0.92, p=$ .817 ; novel present, high uncertainty: $\bar{x}=-0.01, t(33)=-1.25, p=.89$; Figure 3D).

There was no evidence of forced fusion regardless of novel cue presence in the matching patch or level of uncertainty

If the novel shape cue was subject to forced fusion with familiar shape cues to colour, then we expect thresholds in the forced fusion block to be higher when the novel shape cue is present, as the conflicting shape information would make the test patch look more like the target patch than when the judgement is made based on familiar cues alone (when the novel shape cue is absent). Conversely, if novel and familiar cues are not subject to forced fusion the conflicting shape information can be ignored, and thresholds should be the same regardless of novel cue presence in all conditions. Figures $3 \mathrm{E}$ and $3 \mathrm{~F}$ suggest that thresholds were not increased when the novel cue was present regardless of conflict direction and regardless of level of uncertainty. Indeed, a 2 (conflict direction: $\mathrm{C}+\mathrm{S}$ - or $\mathrm{C}-\mathrm{S}+$ ) $\times 2$ (novel cue presence: present or absent) $\times 2$ (uncertainty: low or high) repeated measures ANOVA finds no three-way interaction effect $(F(1,28)=0.76, p=.340)$, no two-way interaction effect of cue presence and either of the other factors (cue presence $x$ conflict direction: $F(1,28)=0.14, p=.713$; cue presence $\mathrm{x}$ uncertainty: $F(1,28)=2.15, p=.015)$, and no main effect of cue presence $(F(1,28)=0.80, p=.378)$. There was a main effect of uncertainty with significantly higher thresholds in the high uncertainty condition $(F(1,28)=35.98, p<.001)$. 


\section{Interim Discussion}

In Experiment 1, we found weak evidence for reliability-weighted averaging. In the low uncertainty condition, variability was reduced when the novel cue was present in the matching patch suggesting participants could combine the novel and familiar cues to enhance perception. However, there was no reduction in variable error when the novel cue was present in the matching patch in the high uncertainty condition and the inferred weight placed on the novel cue was not significantly different to zero in any condition.

We also found mixed evidence for a perceptual effect of the novel cue. In the low uncertainty condition, where participants made simultaneous matches and all information (novel and familiar cues) was present on the screen regardless of whether the novel cue was present in the matching patch, matching variability was lower when the novel cue was present in the matching patch. Recall that in this simultaneous matching condition, regardless of whether the novel cue is present or absent in the matching patch, the same information is available to the participants to complete the task (both familiar and novel cues to colour are present on the screen). Thus, we propose this result points towards a perceptual effect of the novel cue, as there was a reduction in matching variability when the novel shape cue was present in the matching patch but not when it was absent, even though the same information is available in the reference patch when the novel cue is absent from the matching patch. However, we did not find any evidence of a perceptual effect in the forced fusion task.

It is possible that we only saw a reduction in variability in the low and not high uncertainty condition as the effect is small and data in the high uncertainty condition is noisier than in the low uncertainty condition, making the effect harder to detect. Similarly, the fact that weight on the novel cue was not significantly different to zero when cues were conflicting suggests that the reliabilities of the two cues are mismatched, with the familiar cue being much more reliable than the novel shape cue. Thus, it is not surprising that we found no evidence of forced fusion, as little weight would be placed on the conflicting cue. These initial results are therefore somewhat inconclusive, possibly with reasons to do with the reliabilities of the two cues. We addressed these issues in Experiment 2.

\section{Experiment 2}

In Experiment 2, we decreased the reliability of the familiar cue to better match the reliability of the novel cue and encourage participants to place more weight on the novel cue. We decreased familiar cue reliability by adding chromatic noise to the reference patch and having a delay between the presentation of the reference and matching patch in both high and low uncertainty conditions. We 
again tested for a perceptual effect of the novel cue, but in a different way that did not require cue reliabilities to be well matched. Here, we tested for a perceptual effect by testing for a memory colour effect (Witzel \& Hansen, 2015), such as when a grey banana appears slightly yellow (Hansen et al., 2006; Olkkonen et al., 2008; Witzel et al., 2011). Hansen et al. (2006) illustrated this effect by asking participants to adjust the colour inside the outline of a banana until it appeared grey, showing that the grey match made inside the banana shape was bluer than a control match to grey. This suggests that participants had to compensate for the banana appearing yellow (the memory colour effect) by adding blue to the match. This literature suggests that this is a promising domain in which to look for perceptual effects of a newly-learned novel shape cue to colour as the familiar shape of an object has been shown to affect colour appearance.

\section{Methods}

\section{Participants}

We recruited 45 participants (30 female, age range 18-32 years) to take part in Experiment 2. Fifteen participants were excluded as they failed to meet our inclusion criteria (see below). Participants were recruited through word of mouth, our department's undergraduate participant pool, and our lab's Facebook page. Before they could take part in the experiment, participants were screened for colour vision deficiencies using the Ishihara plates. All participants were compensated with $f 10$ for their time plus an additional bonus of $£ 5-10$ depending on performance. If participants got $X \%$ of the total points available, they received $f(X+10) / 10$ bonus, rounded down, but received a minimum of f5.

Ethics

Ethical approval was received from the Durham University Psychology Department Ethics Board (reference number: 17/07). All participants gave written, informed consent prior to taking part in the experiment.

\section{Apparatus}

We used the same apparatus as that used for Experiment 1.

Stimuli

We used the same gradient of colours and the same mapping between colours and axis ratios as in Experiment 1.

To decrease familiar cue reliability, we added chromatic noise to the reference stimulus (Figure 4). To add chromatic noise, reference patches were filled with a checkerboard pattern with each check 
on the board filled with either the true reference colour, or the true reference colour plus or minus a small jitter. The jitter was 0.03 of the colour gradient in the low chromatic noise condition and 0.1 of the colour gradient in the high chromatic noise condition. How the checkerboard was filled was pseudo-randomised to ensure that the mean colour was with $1.5 \Delta E$ of the true reference colour in CIELUV.

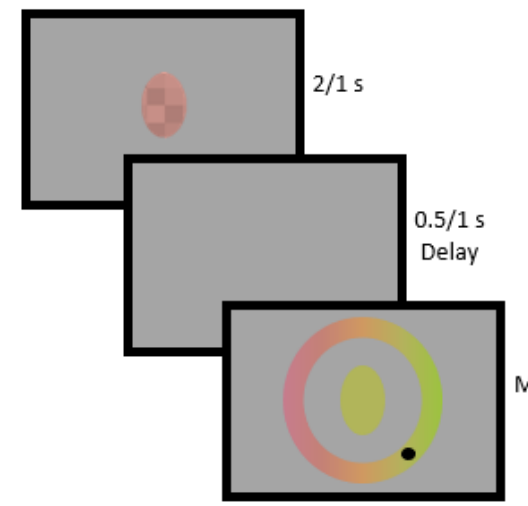

No Conflict, Low/High Uncertainty, Novel Cue Present in Matching Patch

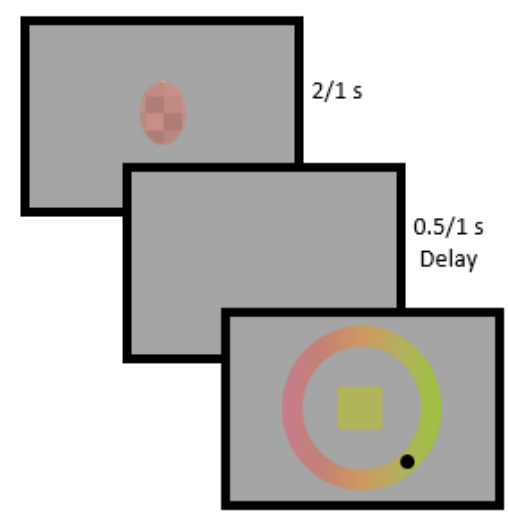

No Conflict, Low/High Uncertainty, Novel Cue Absent in Matching Patch

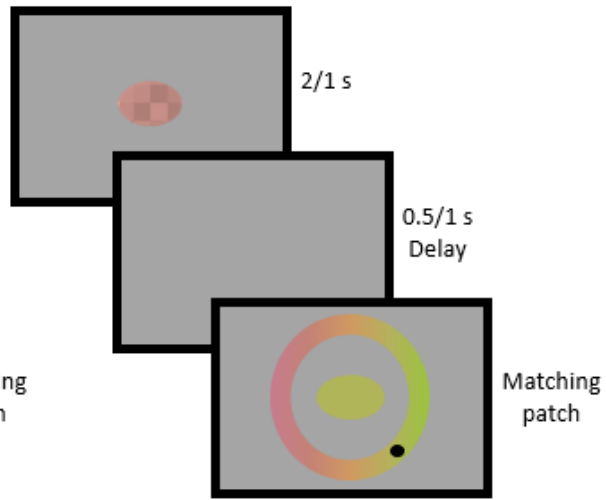

Conflict, Low/High Uncertainty, Novel Cue Present in Matching Patch

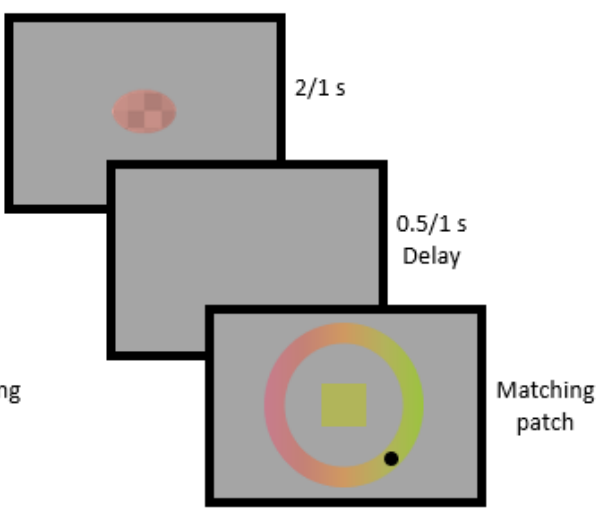

Conflict, Low/High Uncertainty, Novel Cue Absent in Matching Patch

Figure 4: Adjustment trials in the test blocks of Experiment 2. There were two blocks of test trials in Experiment 2, one with low uncertainty (a reference patch presentation time of 2 seconds and a delay of 0.5 seconds before presentation of the matching patch) and one with high uncertainty (a reference patch presentation time of 1 second and a delay of 1 second before presentation of the matching patch). In each test block trials were one of four types, varying whether the novel shape cue was present in the matching patch and whether the novel shape cue conflicted with the colour of the reference patch according to the novel relationship introduced in a preceding exposure block.

\section{Procedure}

There were four main blocks of trials in the task: the exposure block (block 1), the low uncertainty test block (block 2), the top-up exposure block (block 3), and the high uncertainty test block (block 4). Blocks 2 and 4 were switched at random for each participant. Interleaved with the four main blocks of trials were sets of achromatic matches. There was a control set of achromatic matches at 
the start of the experiment and a test set of achromatic matches at the end of each block of trials resulting in five sets of achromatic matches in total.

Blocks 1 to 4 consisted of adjustment trials, where participants could adjust the colour of one patch (the matching patch) to match the colour of another patch (the reference patch). There were eight types of adjustment trials as the shape of the matching patch, the noise in the reference patch and delay between the reference and matching patch, and whether the familiar and novel cues were in conflict could vary. As in Experiment 1, these manipulations addressed our research questions. The shape of the reference patch (oval or square) manipulated the presence of the novel shape cue in the matching patch (novel cue present or absent). The noise in the reference patch, duration of the reference patch, and delay between presentation of the reference and matching patch (low noise/2 second duration/500 millisecond delay or high noise/1 second duration/1 second delay) manipulated the uncertainty of familiar (low-level) cues to colour (low or high familiar cue uncertainty). Adding conflict between the familiar cues to colour and novel shape cue to colour allowed us to measure the amount of weight that participants placed on the novel cue.

During the exposure block adjustment trials were all the same type: no conflict, low uncertainty trials with the novel cue present in the matching patch. Participants were first presented with the reference patch for 2 seconds and the matching patch 500 milliseconds later (Figure 4). The mean colour of the reference patch had a value between 0.1 and 0.9 in steps of 0.05 , each shown 11 times - 187 trials in total. We had to reduce the range of stimuli shown in Experiment 2 (compared to Experiment 1 ) to allow for variation of the colour in the reference patch (the addition of chromatic noise). Both the reference and matching patch were ovals with the axis ratio that corresponded to the colour value of the reference patch. The starting colour of the matching patch was a random value between 0 and 1 . Around the matching patch, participants saw the whole colour gradient as a ring. By moving the mouse, they could move a black circle around the ring, changing the colour of the matching patch, and left click to issue a response. After responding, participants received feedback in the form of points for that trial. Points were awarded according to a mean squared error loss function.

Adjustment trials in the low uncertainty test block were the same as in the exposure block, except that the matching patch could be a square on some trials and/or the axis ratio of the oval could conflict with the corresponding colour (Figure 4). Thus, there were four types of trials in this block: (1) no conflict, low uncertainty trials with the novel cue present in the matching patch; (2) no conflict, low uncertainty trials with the novel cue absent in the matching patch; (3) conflicting, low uncertainty trials with the novel cue present in the matching patch; (4) conflicting, low uncertainty 
trials with the novel cue absent in the matching patch. For trials where there was no conflict, regardless of whether the novel cue was present or absent in the matching patch, the mean colour of the reference patch had an arbitrary value between 0.1 and 0.9 in steps of 0.1 , each repeated 5 times for each trial type. In trials without conflict and where the novel cue was present in the matching patch, the reference and matching shape were ovals with axis ratios corresponding to the colour of the reference patch. In trials without conflict and where the novel cue was absent from the matching patch, the matching patch was a square with side length $5.2 \%$ of the screen width. For the trials with conflict, regardless of whether the novel cue was present or absent in the matching patch, the mean colour of the reference patch had an arbitrary value of 0.25 or 0.75 , but the axis ratio of the reference oval conflicted with this value by $-10,-5,5$, or $10 \%$ of the mapping. In trials with conflict and where the novel cue was present in the matching patch, the matching patch was also an oval with the conflicting value. In trials with conflict and where the novel cue was absent from the matching patch, the matching patch was a square with side length $5.2 \%$ of the screen width. There were five repeats of each reference and conflict value pairing for each trial type and a total of 170 trials in this block in total.

The top-up exposure block was identical to the exposure block, containing only no conflict, low uncertainty trials with the novel cue present in the matching patch, except that the number of repeats for each reference was reduced to 4 for 68 trials in this block in total.

The high uncertainty test block was identical to the low uncertainty test block except that the reference patch was now only shown for 1 second, and participants were only allowed to issue their response after a 1 second delay (Figure 4). Hence, this block also contained four types of trials: (1) no conflict, high uncertainty trials with the novel cue present in the matching patch; (2) no conflict, high uncertainty trials with the novel cue absent in the matching patch; (3) conflicting, high uncertainty trials with the novel cue present in the matching patch; (4) conflicting, high uncertainty trials with the novel cue absent in the matching patch.

The control set of achromatic matches was designed to measure a control match to grey for each participant. This set of matches was at the start of the experiment before any exposure to the novel shape-colour association. Participants made five matches to grey with the shape varying (arbitrary values of $0.1,0.3,0.5,0.7,0.9$ ) and the order of the shapes randomised. Participants were instructed to "adjust the patch so that it appears grey (neither blue, yellow, red, or green)". They made the adjustment using the left, right, down, and up arrow keys on the keyboard. Each key changed the colour by $0.25 \Delta E$ in CIELUV colour space. The up key increased the value along the $v^{*}$ axis, the 
down key decreased the value along the $v^{*}$ axis, the right key increased the value along the $u^{*}$ axis, and the left key decreased the value along the $u^{*}$ axis.

In each subsequent set of achromatic matches, participants did the same as for the control set of achromatic matches, except that there were 3 repeats of each shape in a random order.

\section{Data Analysis}

Data from the adjustment trials was analysed in the same way as in Experiment 1.

To analyse the achromatic matches we calculated memory colour indices (Figure 5). Memory colour indices were calculated separately for each test shape and each set of achromatic matches. For test shape $\mathrm{X}$ in achromatic match set $\mathrm{Y}$, we calculated the memory colour index as

$$
m=\frac{|\overrightarrow{\boldsymbol{a}}|}{|\overrightarrow{\boldsymbol{b}}|}=\frac{\overrightarrow{\boldsymbol{b}} \cdot-\overrightarrow{\boldsymbol{t}}}{|\overrightarrow{\boldsymbol{b}}|^{2}}
$$

where $\overrightarrow{\boldsymbol{t}}$ is the vector between the mean control match (the mean over the five matches in the control set of achromatic matches) and the mean shape $X$ match in set $Y, \vec{b}$ is the vector between the mean shape $X$ match in set $Y$ and the colour of the shape according to the novel mapping, and $\vec{a}$ is the vector projection of $-\overrightarrow{\boldsymbol{t}}$ on $\overrightarrow{\boldsymbol{b}}$. Subsequently, a positive colour memory index indicates the presence of the classic colour memory effect, where achromatic matches are pushed away from the associated shape colour towards the opposing hue. A larger positive colour memory index indicates a larger memory colour effect. A negative colour memory index indicates the opposite of the classic colour memory effect, where achromatic matches are pulled towards the associated shape colour. A smaller negative colour memory index indicates a larger opposite effect.

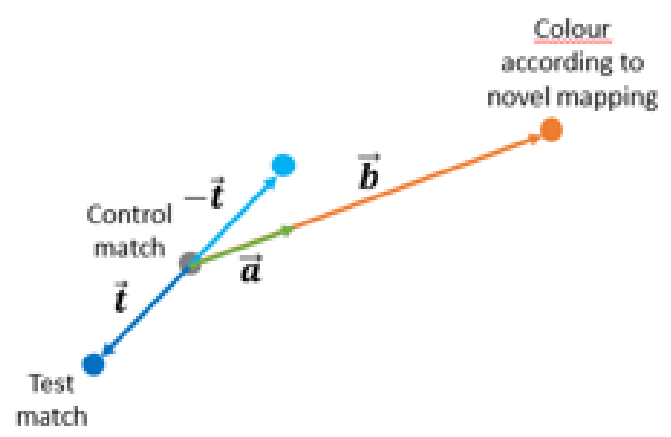

\section{Memory colour index}

$$
m=\frac{|\vec{a}|}{|\vec{b}|}=\frac{\vec{b} \cdot-\vec{t}}{|\vec{b}|^{2}}
$$

Figure 5: Calculating memory colour indices.

Exclusion Criteria 
As in Experiment 1, we planned to exclude participants from the analyses if their matching variability was too high for either of the low or high uncertainty conditions. Matching variability had to be less than 0.075 for the low uncertainty condition and less than 0.15 for the high uncertainty condition.

\section{Results}

Matching variability reduced when the novel shape cue was present in the matching patch but only in the low uncertainty condition

As in Experiment 1, we found significantly lower matching variability when the novel cue was present in the low uncertainty condition ( $z=-2.07, p=.039)$, but no difference between when the novel cue was present and when it was absent in the high uncertainty condition $(z=0.38, p=$ $.704)$. 
A

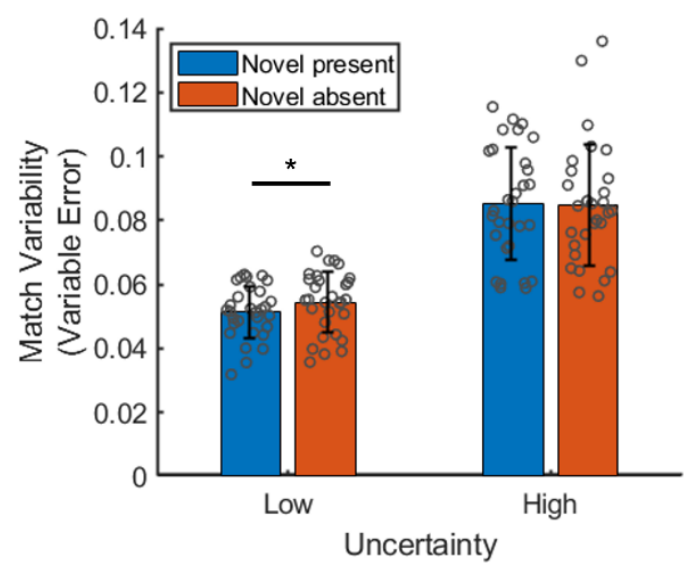

B

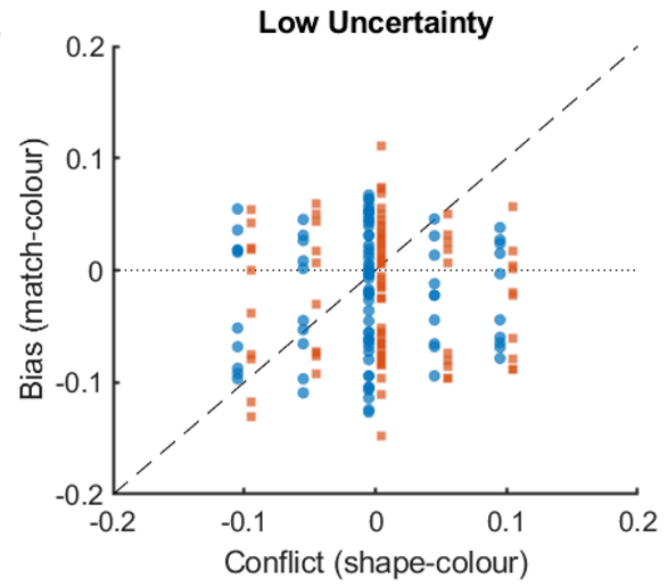

C

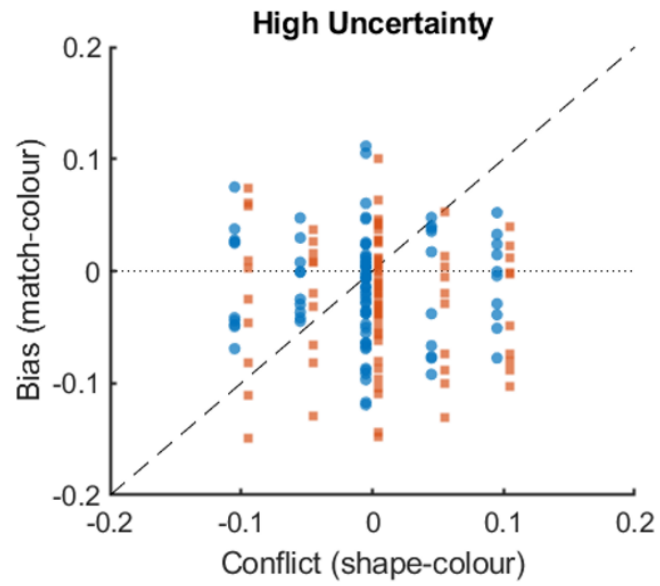

D

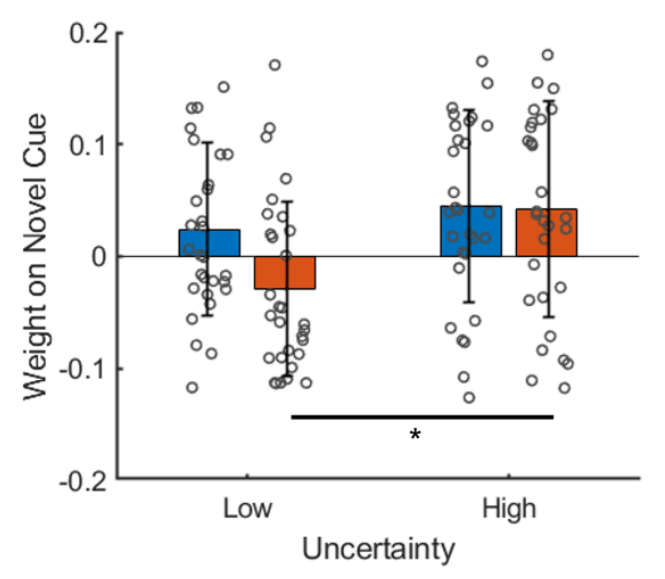

E

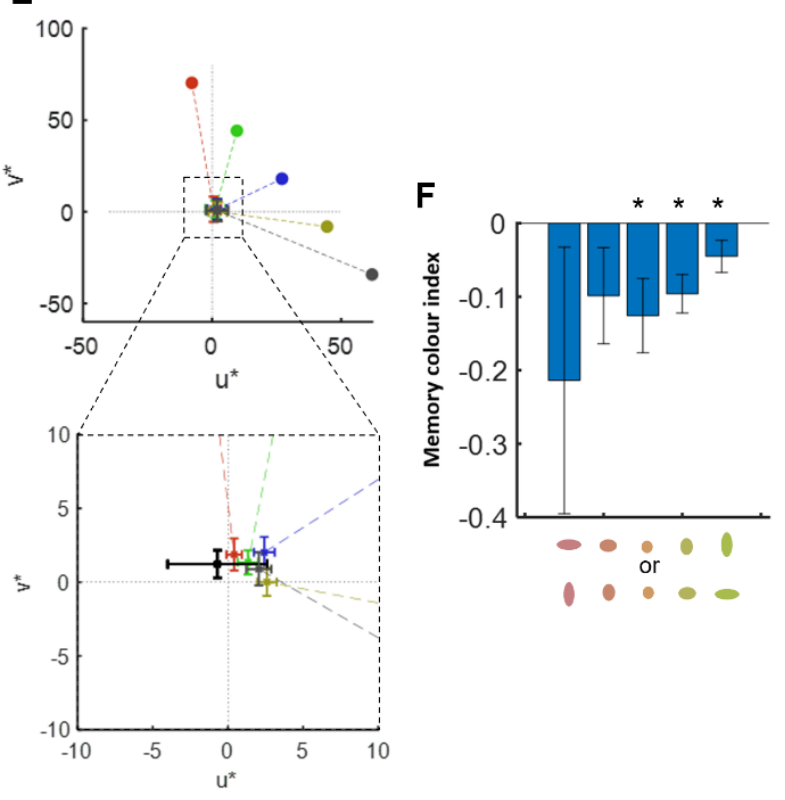

Figure 6: Experiment 2 results. (A) Matching variability (variable error) for each level of uncertainty split by novel cue presence. $(B / C)$ Individual matching biases (matched colour - reference colour) for an example participant plotted against the conflict between the shape (novel) and colour (familiar) cues in the reference patch (shape cue value - colour cue value) in the low $(B)$ and high $(C)$ uncertainty conditions. Blue dots are when the novel cue was present in the matching patch. Orange squares are when the novel cue was absent from the matching patch. (D) The inferred weight on the novel cue in 
each condition. (E) Example raw colour matches from the fourth set and the calculated memory colour indices. Error bars are +/- $1 S D$.

When novel and familiar cues conflicted, the weight placed on novel cue was flexible

As we did for Experiment 1, in Figures $6 \mathrm{~B}$ and $6 \mathrm{C}$ we show data from an example participant on trials where the novel shape cue conflicted with familiar cues to colour. Much like in Experiment 1, these figures suggest that conflicting novel shape cues have little effect on the bias in colour matches. However, averaging across all participants (Figure 6D), there was a small but significant positive weight on the novel cue in all conditions except when uncertainty was low and the novel cue was absent from the matching patch (novel absent, low uncertainty: $\bar{x}=-0.03, t(29)=-2.06, p=$ .976; novel absent, high uncertainty: $\bar{x}=0.04, t(29)=2.37, p=.012$; novel present, low uncertainty: $\bar{x}=0.02, t(29)=1.7, p=.05$; novel present, high uncertainty: $\bar{x}=0.04, t(29)=$ $2.87, p=.004)$. Under the assumption of reliability-weighted averaging, we expected the weight on the novel cue to increase with increasing uncertainty. This was true when the novel cue was absent from the matching patch (significantly greater weight in the high condition, $t(29)=$ $3.79, p<.001$; compare orange bars in Figure $6 \mathrm{D}$ ), but not when the novel cue was present in the matching patch $(t(29)=0.97, p=.171$; compare blue bars in Figure $6 \mathrm{D})$.

\section{There was no evidence of a memory colour effect}

As an example, Figure 6E shows the mean achromatic match for each colour tested in the fourth and final set of achromatic matches. The inset enlarges the area immediately surrounding the matches where the control match (black error bars) can also be seen. It is clear from this figure that all matches are shifted away from the control match. To quantify this effect, we calculated memory colour indices (see data analysis section). In a 5 (colour) x 4 (match set) repeated measures ANOVA there was no interaction effect of match set and colour $(F(12,336)=0.96, p=.486)$ and no main effect of match set $(F(3,84)=0.86, p=.466)$ so we collapsed memory colour indices over matching sets to get a mean memory colour index for each set of matches (Figure 6F). The mean memory colour index was consistently less than zero for all five colours tested but only significantly so for three of them $(0.1: m=-0.21, t(28)=-1.18, p=.124 ; 0.3: m=-0.1, t(28)=$ $-1.51, p=.071 ; 0.5: m=-0.13, t(28)=-2.49, p=.009 ; 0.7: m=-0.1, t(28)=-3.67, p<$ $.001 ; 0.9: m=-0.05, t(28)=-2.06, p=.024)$. In other words, there was a significant effect in the opposite direction of a classic memory colour effect for three of the colours tested.

\section{General Discussion}


We extend the limited work on efficient use of novel cues to the domain of material perception. We tested participants in a short-term training study with a novel shape-colour association, making object shape a useful cue to object colour within the task. We tested for two signatures of reliabilityweighted averaging of the novel shape cue with familiar cues to colour (a variability reduction and cue re-weighting) and for a perceptual effect of the novel cue. We found signatures of reliabilityweighted averaging of the novel shape cue and familiar cues to colour in both experiments we presented. Participants showed a reduction in colour matching variability in both experiments and cue reweighting with changes in uncertainty in Experiment 2. However, our results overall suggest that the ability to quickly learn novel cues and take a reliability-weighted average of novel and familiar cues is not immediately accompanied by common perceptual effects.

In Experiment 1, we found weak evidence for reliability-weighted averaging. There was a variability reduction when the novel cue was present in the matching patch, but only in the low uncertainty condition, and the weight placed on the novel cue was not significantly different to zero in any condition. We also found mixed evidence of a perceptual effect of the novel shape cue. In simultaneous matches, matching variability was lower when the novel cue was present in the matching patch, which we interpret as evidence for a perceptual effect of the novel cue, as there was a reduction in matching variability when the novel shape cue was present in the matching patch but not when it was absent, even though the same information is available in the reference patch when the novel cue is absent from the matching patch. However, there was no evidence of a perceptual effect from the forced fusion task.

We proposed that the level of mismatch between cue reliabilities in Experiment 1 could have hindered our ability to detect the effects we were looking for and rectified this in Experiment 2 by making familiar cues to colour more uncertain and better matched in reliability to the novel shape cue to colour. As intended, this manipulation encouraged participants to place more weight on the novel shape cue in Experiment 2, where the weight placed on the novel cue was significantly greater than zero in three of four conditions. However, there was only a significant increase in the weight on the novel cue with increased familiar cue uncertainty when the novel cue was absent from the matching patch. We also tested for a perceptual effect of the novel cue in Experiment 2 using a method that did not require well-matched cue reliabilities by testing for a memory colour effect. For three of the five colours tested, we found a significant effect in the opposing direction to the classic memory colour effect.

Our results add to the growing literature showing that novel cues can be learned and integrated with familiar cues to enhance perception (Aston, Beierholm, et al., 2021; Ernst, 2007; Gibo et al., 2017; 
Negen et al., 2018, 2021) and extend this finding to the domain of material perception. We have expanded upon these previous findings by testing for a perceptual effect of the novel cue to colour that we introduced. In Experiment 1, we found some evidence of a perceptual effect, as matching variability was lower when the novel cue was present in the matching patch, but no evidence of forced fusion. In Experiment 2, we found a significant memory colour effect for three of four colours tested, but it was in the opposite direction to a classic memory colour effect.

An inability to acquire memory colour effects from novel shape-colour associations has been reported elsewhere. In a book chapter, Witzel and Hansen (2015) summarised some of their own unpublished work and the work of others (Huebner and Giesel) obtained through personal communication. In these works, participants were taught novel shape-colour associations and the experimenters tested for a memory colour effect. Witzel and Hansen (2015) reported that neither study found any evidence of an acquired memory colour effect. They did not report how long participants were trained with the novel associations, but it is likely that the exposure periods were short, as in our experiment, and that longer training periods are required before shape becomes diagnostic of an object's colour and induces a memory colour effect. In unpublished work, Karl Gegenfurtner and colleagues (personal communication) trained new colour-shape associations (red triangles and blue squares) for at least 10 hours in one lab-based study and gave people woollen balls to take home and carry around with them for a few weeks in another study. They also found no evidence of a memory colour effect in either study. It is possible that only certain object colours lead to a memory colour effect. Witzel and Hansen (2015) hypothesised that memory colour effects are strongest for objects with diagnostic colours falling on the daylight axis (the axis that maps out changes in daylight chromaticities across the day - blue to yellow) as achromatic adjustments are more uncertain along this axis. Indeed, Olkkonen et al. (2008) found the largest memory colour effect for a banana (yellow) and the smallest memory colour effect for a strawberry (red). The former is a colour that lies on the daylight axis, while the latter lies on a line perpendicular to the daylight axis in the CIELUV uniform colour space. As our colours varied from pinkish to greenish, they may not be the optimal colours to use for inducing a memory colour effect. Future studies may find different results using a blue-yellow colour gradient.

Why, though, did we observer the opposite of a memory colour effect? A simple interpretation is that participants in our experiment did something different to what was asked of them and matched the patches to the associated colour rather than grey. However, that seems unlikely given the size of the memory colour indices, the matches were only slightly (but consistently) pulled towards the associated colour. Another possibility is that adaptation played a role. All shapes seen in our experiment came from a colour gradient that was defined as a chord along a hue circle in CIELUV. It 
is possible that participants adapted to the gradient of colours so that they could not see the slight colour left in their grey matches. However, both suggestions are very speculative and further work is needed to explore the opposite effects.

It is possible that we did not see any evidence for the familiar perceptual effects we tested for in our experiments as our participants were only exposed to the novel shape-colour association for a short time. Other authors have suggested that the reason children do not combine multiple familiar cues is because cues are still being cross-calibrated throughout development, and that cues are not combined until cross-calibration is complete to retain access to individual cues (Gori et al., 2008). A similar explanation could account for participants showing only weak evidence of combination in the experiments presented here. Future experiments should explore the effect of longer-term training.

\section{Conclusions}

In two experiments observers showed signatures of reliability-weighted averaging of a newlylearned novel shape cue to colour with familiar cues. Specifically, they showed a precisionimprovement in both experiments and reweighting of the newly-learned shape cue with changes in uncertainty in Experiment 2. However, we find no evidence that reliability-weighted averaging of novel and familiar cues is accompanied by perceptual effects, finding no evidence for forced fusion in Experiment 1 and the opposite of a memory colour effect in Experiment 2. These results suggest that while people rapidly learn new statistical regularities to optimise their perceptual decisions, these are not immediately (with short training) accompanied by familiar perceptual effects. How much experience is needed to see familiar perceptual effects with newly learned cues is an open question for future research.

\section{Acknowledgements}

We would like to thank Anya Hurlbert for use of the Konica Minolta CS2000 and James Negen for many useful conversations. This project has received funding from the European Research Council (ERC) under the European Union's Horizon 2020 research and innovation programme (grant agreement No. 820185) and a Leverhulme Trust Research Project Grant (RPG-2017-097).

\section{Supporting Information Online}

Raw data and analysis scripts are available on OSF: https://osf.io/ikuvr/.

\section{References}

Aston, S., Beierholm, U., \& Nardini, M. (2021). Newly learned novel cues to location are combined with familiar cues but not always with each other. PsyArXiv, 1-32. 
Aston, S., Negen, J., Nardini, M., \& Beierholm, U. (2021). Central tendency biases must be accounted for to consistently capture Bayesian cue combination in continuous response data. Behavior Research Methods. https://doi.org/10.3758/s13428-021-01633-2

Brainard, D. H. (1997). The Psychophysics Toolbox. Spatial Vision, 10(4), 10:433-436. http://www.ncbi.nlm.nih.gov/pubmed/9176952

Ernst, M. O. (2007). Learning to integrate arbitrary signals from vision and touch. Journal of Vision, 7(5), 7. https://doi.org/10.1167/7.5.7

Ernst, M. O., \& Banks, M. S. (2002). Humans integrate visual and haptic information in a statistically optimal fashion. Nature, 415(6870), 429-433. https://doi.org/10.1038/415429a

Gibo, T. L., Mugge, W., \& Abbink, D. A. (2017). Trust in haptic assistance: weighting visual and haptic cues based on error history. Experimental Brain Research, 235(8), 2533-2546. https://doi.org/10.1007/s00221-017-4986-4

Gori, M., Del Viva, M., Sandini, G., \& Burr, D. C. (2008). Young Children Do Not Integrate Visual and Haptic Form Information. Current Biology, 18(9), 694-698. https://doi.org/10.1016/j.cub.2008.04.036

Hansen, T., Olkkonen, M., Walter, S., \& Gegenfurtner, K. R. (2006). Memory modulates color appearance. Nature Neuroscience, 9(11), 1367-1368. https://doi.org/10.1038/nn1794

Hillis, J. M., Ernst, M. O., Banks, M. S., \& Landy, M. S. (2002). Combining Sensory Information: Mandatory Fusion Within, but Not Between, Senses. Science, 298(5598), 1627-1630. https://doi.org/10.1126/science.1075396

Hillis, J. M., Watt, S. J., Landy, M. S., \& Banks, M. S. (2004). Slant from texture and disparity cues: Optimal cue combination. Journal of Vision, 4(12), 1. https://doi.org/10.1167/4.12.1

Jacobs, R. A. (1999). Optimal integration of texture and motion cues to depth. Vision Research, 39(21), 3621-3629. https://doi.org/https://doi.org/10.1016/S0042-6989(99)00088-7

Jones, P. R. (2016a). A tutorial on cue combination and Signal Detection Theory: Using changes in sensitivity to evaluate how observers integrate sensory information. Journal of Mathematical Psychology, 73, 117-139. https://doi.org/https://doi.org/10.1016/j.jmp.2016.04.006

Jones, P. R. (2016b). A tutorial on cue combination and Signal Detection Theory: Using changes in sensitivity to evaluate how observers integrate sensory information. Journal of Mathematical Psychology, 73, 117-139. https://doi.org/https://doi.org/10.1016/j.jmp.2016.04.006 
Kleiner, M., Brainard, D. H., \& Pelli, D. (2007). What's new in Psychtoolbox-3? Perception 36 ECVP Abstract Supplement.

Knill, D. C., \& Saunders, J. A. (2003). Do humans optimally integrate stereo and texture information for judgments of surface slant? Vision Research, 43(24), 2539-2558. https://doi.org/10.1016/S0042-6989(03)00458-9

Landy, M. S., Banks, M. S., \& Knill, D. C. (2011). Ideal-observer models of cue integration. In Sensory cue integration (pp. 5-29). Oxford University Press.

Nardini, M., Bedford, R., \& Mareschal, D. (2010). Fusion of visual cues is not mandatory in children. Proceedings of the National Academy of Sciences, 107(39), 17041-17046. https://doi.org/10.1073/pnas.1001699107

Negen, J., Bird, L.-A., Slater, H., Thaler, L., \& Nardini, M. (2021). A New Sensory Skill Shows Automaticity and Integration Features in Multisensory Interactions. BioRxiv, 2021.01.05.425430. https://doi.org/10.1101/2021.01.05.425430

Negen, J., Wen, L., Thaler, L., \& Nardini, M. (2018). Bayes-Like Integration of a New Sensory Skill with Vision. Scientific Reports, 8(1), 16880. https://doi.org/10.1038/s41598-018-35046-7

Olkkonen, M., Hansen, T., \& Gegenfurtner, K. R. (2008). Color appearance of familiar objects: Effects of object shape, texture, and illumination changes. Journal of Vision, 8(5), 13. https://doi.org/10.1167/8.5.13

Pelli, D. G. (1997). The VideoToolbox software for visual psychophysics: Transforming numbers into movies. Spatial Vision, 10, 10:437-442.

Plummer, M. (2003). JAGS: A Program for Analysis of Bayesian Graphical Models using Gibbs Sampling. 3rd International Workshop on Distributed Statistical Computing (DSC 2003); Vienna, Austria, 124.

Rohde, M., van Dam, L. C. J., \& Ernst, M. O. (2016a). Statistically Optimal Multisensory Cue Integration: A Practical Tutorial. Multisensory Research, 29(4-5), 279-317. https://doi.org/https://doi.org/10.1163/22134808-00002510

Rohde, M., van Dam, L. C. J., \& Ernst, M. O. (2016b). Statistically Optimal Multisensory Cue Integration: A Practical Tutorial. Multisensory Research, 29(4-5), 279-317. https://doi.org/https://doi.org/10.1163/22134808-00002510

Witzel, C., \& Hansen, T. (2015). Memory effects on color perception. In A. J. Elliot, A. Franklin, \& M. 
D. Fairchild (Eds.), Handbook of Color Psychology (pp. 641-659). Cambridge University Press. https://doi.org/DOI: 10.1017/CBO9781107337930.032

Witzel, C., Valkova, H., Hansen, T., \& Gegenfurtner, K. R. (2011). Object knowledge modulates colour appearance. I-Perception, 2, 13-49. 